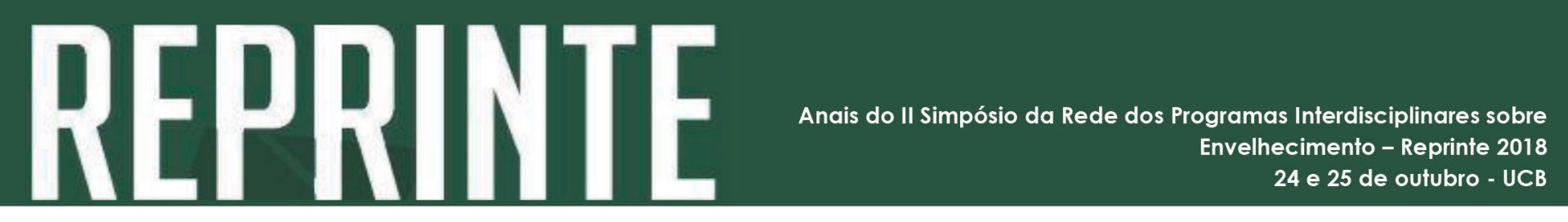

http://dx.doi.org/10.5335/rbceh.v16i1.9764

\title{
10) Cartografia da saúde de idosos atendidos em unidades básicas de saúde pública
}

Danieli Tefili Rossa'; Eduardo dos Santos de Lima'; Silvana Alba Scortegagna²

\section{Resumo}

Com o envelhecimento populacional pode ocorrer aumento da incidência de doenças crônicas e procura de assistência à saúde. Objetivou-se com este estudo verificar as condições de saúde de idosos atendidos em unidades de saúde pública da cidade de Getúlio Vargas-RS. Foram coletados dados de diagnóstico nos relatórios de atendimento (e-SUS) de todas Unidade Básica de Saúde (UBS, n=5), no período de 01 de janeiro à 30 de julho de 2018. Levantaram-se 8.634 consultas, 8.524 nas UBS e 110 à domicílio. Observou-se a prevalência da Classificação Internacional de Doenças (CID) Exame Médico Geral ( $Z=000, n=1.144)$, Hipertensão Essencial primária (I10, n=439) e Exames de Pressão Arterial (Z013, n=169). A prevalência do atendimento ocorreu em mulheres $(n=5.309)$ na faixa etária de 60 a 64 anos. Atenção especial deve ser dada às consultas de rotinas e à elevada incidência de doenças crônicas relacionadas à pressão arterial, especialmente em mulheres, nas políticas públicas de cuidado a saúde.

Palavras-Chave: Diagnóstico; Relatório; Saúde Pública; Idosos; Doenças.

\section{Os idosos atendidos nas UBS do município de Getúlio Vargas (RS)}

Com a crescente expectativa de vida e baixa natalidade faz-se a transição demográfica. Pessoas com 60 anos ou mais, consideradas idosas pela OMS (Organização Mundial da Saúde - OMS, 2015) poderão chegar atingir a longevidade. Nesse cenário, o Brasil supera a marca de 30,2 milhões de idosos, com um número de mulheres de 56\% e homens de 44\% (OMS, 2015). Esse número crescente apresenta consistência em todas as regiões do país. O Rio Grande do Sul, especificamente, é um dos estados com maior proporção de idosos (IBGE, 2018).

Pela vulnerabilidade dessa etapa da vida, podem surgir reduções progressivas das capacidades fisiológica e funcionais do organismo, o que pode colocar em risco a saúde física-mental e trazer problemas sociais para estes indivíduos. Para Santos et al (2018) as pessoas idosas

Mestrandos no Programa de Pós-Graduação em Ciência do Envelhecimento Humano. Endereço para correspondência: Universidade de Passo Fundo-UPF, BR 285, São José I Passo Fundo/RS I CEP: 99052-900. Emails (na ordem em que aparecem): danitefili@gmail.com; eduardo_sevn@hotmail.com

2 Doutora em Psicologia pela Universidade São Francisco. Professora do Programa de Pós-Graduação Stricto Sensu em Envelhecimento Humano da Universidade de Passo Fundo. E-mail: silvanalba@upf.br 
estão configurando um novo perfil demográfico, trazendo vulnerabilidades e incapacidades associadas ao passar dos anos, juntamente com a alteração do sistema imunológico, diretamente relacionado ao surgimento de doenças e às comorbidades (AGONDI et al, 2012). O trabalho de prevenção e cuidado a saúde pode fazer com que as doenças comumente associadas ao envelhecimento não ganhem tanta expressão, oferecendo benefícios a esse grupo.

Alguns avanços na área da saúde pública brasileira demonstram intervenções relacionadas à prevenção e ao tratamento de patologias, ao direito universal e integral à saúde, descritos no ministério da Saúde (BRASIL, 1990). Um resultante disso é a elevada procura pelos serviços de saúde pública. Os municípios possuem as UBS, onde atendem pelo SUS, mantido pelo próprio município com ajuda do governo do estado, desenvolvendo auxílios para a promoção da saúde. Neste cenário, o município de Getúlio Vargas, situado no norte do Rio Grande do Sul, possui 16.154 habitantes e conta com 5 UBS, com diversas especialidades, onde se atendem pessoas de todas as idades. Diante da necessidade de contribuir para o direcionamento de ações de programas de saúde preventiva, esse estudo buscou verificar as condições de saúde de idosos atendidos nas UBS.

\section{Método}

Estudo transversal, de base populacional, realizado a partir de análise do relatório e-SUS vinculado ao Ministério da Saúde do Estado do Rio Grande do Sul, do município de Getúlio Vargas, no período de 01 de janeiro de 2018 até 30 de julho de 2018 (6 meses), de pessoas com 60 anos de idade e mais.

Para responder ao objetivo proposto, realizou-se um mapeamento nos relatórios das UBS (e-SUS), focalizando o CID emitido, a quantidade de idosos que procuraram atendimento, idade, gênero e local onde o atendimento foi efetivado.

\section{Resultados}

Os idosos, com 60 anos de idade e mais, usuários da saúde pública da cidade de Getúlio Vargas - RS, somaram 8.634 consultas, nos últimos 6 meses. Dessas 8.524 foram realizadas nas UBS e 110 foram efetuadas a domicílio. O número de mulheres atendidas foi de 5.309 e o de homens foi de 3.325. Observou-se que a CID mais frequente foi de Exame Médico Geral ( $n=1.144)$, seguido pela Hipertensão Essencial primária $(n=439)$, e a Solicitação de Exames da Pressão Arterial ( $\mathrm{n}=169)$. Na tabela 1 constam os achados referentes ao número de idosos atendidos e CID: 
Tabela 1. Prevalência de CID, nas UBS do município em 2018

\begin{tabular}{llc} 
CID & \multicolumn{1}{c}{ Diagnósticos } & № de idosos \\
Z000 & Exame Médico Geral & 1.144 \\
I10 & Hipertensão Essencial (primária) & 439 \\
Z013 & Exame Pressão Arterial & 169 \\
F411 & Ansiedade Generalizada & 85 \\
E11 & Diabetes Mellitus não-insulino-dependente & 63 \\
M545 & Dor Lombar Baixa & 58 \\
F920 & Depressão & 55 \\
E10 & Diabetes Mellitus insulino-dependente & 41 \\
\hline Fonte própria. &
\end{tabular}

Verifica-se na Tabela 1 a prevalência do CID Z000. Este CID é genérico, usado para consultas de rotina, onde provavelmente a doença deve estar especificada no prontuário médico. Por isso, para fins de relatório, este é um campo que se manteve mais incompleto.

Observa-se, entre os CID com maior frequência, a incidência de grupos semelhantes como no caso da Hipertensão Essencial (primária) e Exame de Pressão Arterial, de Diabetes Mellitus com e sem dependência de insulina e da Ansiedade Generalizada e Depressão. Na tabela 2 encontra-se a distribuição dos idosos conforme gênero, faixa etária e local de atendimento.

Tabela 2. Perfil e Local de Atendimento

$\begin{array}{lc}\text { Indicador } & \text { № de idosos } \\ \text { Gênero } & 5.309 \\ \text { Mulheres } & 3.325 \\ \text { Homens } & \\ \text { Idades } & \\ \text { De } 60 \text { a } 64 & \\ \text { De } 65 \text { a } 69 & \\ \text { De } 70 \text { a } 74 & \\ \text { De } 75 \text { a } 79 & \\ \text { Acima de } 80 & 110 \\ \text { Local de atendimento } & \\ \text { UBS } & \\ \text { Domiciliar } & \end{array}$

Constata-se a predominância de atendimento a mulheres, sendo realizados majoritariamente nas dependências das UBS. Observa-se também que a faixa etária predominante foi de 60 a 64 anos.

\section{Conclusão}

A partir da análise dos relatórios de atendimento de idosos consultados nas UBS do município de Getúlio Vargas - RS, constatou-se a predominância do CID Z000 ( $\mathrm{n}=1.144)$. O fato de ele ser usado de forma genérica torna-o um dado impreciso para ser analisado isoladamen- 
te. Essa informação poderia ser convertida em ações ou programas direcionados a públicos mais específicos. Deve-se ter o cuidado de pensar que um mesmo idoso possa estar inserido repetidas vezes nesses números absolutos, mas, essas possibilidades ainda se oferecem.

Quando as informações são melhor preenchidas, observa-se por exemplo, a incidência de grupos similares. Os CID I10 ( $n=439)$ e Z013 ( $n=169)$ constituem um grande grupo de idosos em que a atenção está nos cuidados à pressão arterial. Essa informação pode ser convertida em ações mais focais, promovendo orientações sobre atividades físicas e alimentação adequadas, promovendo benefícios e práticas mais saudáveis a essa população.

Outro exemplo está nos cuidados em saúde mental dos idosos, Ansiedade Generalizada e Depressão também aparecem nos CID com incidência. Entender esses fenômenos nos idosos e conhecer essa parcela da população da cidade pode servir como base tanto na prevenção quanto na intervenção. Grupos de apoio e suporte e psicoterapia irão compor uma rede de assistência tal qual preconiza as diretrizes do SUS.

Observou-se, ainda, a prevalência de mulheres na busca pelos atendimentos. Um dos motivos para este achado pode estar associado as concepções culturais de que ao homem cabe a responsabilidade de proteger, acreditando não precisar dos cuidados de outras pessoas (BOTTON et al, 2017). Todavia o número de homens idosos em busca de atendimento indica mudanças nessa cultura, movidas também pelas ações de educação em saúde promovidas pelas UBS. Outro fator importante a se levar em conta nessa análise é que a população idosa da cidade tem mais mulheres do que homens (IBGE, 2017).

Os achados mostram prevalência de atendimento nas UBS, mas observou-se, também, grande quantidade de atendimento nos domicílios dos idosos. Percebe-se que os programas de saúde da família que realizam essa intervenção, indo até a casa desses idosos, assume importante papel na promoção à saúde oferecendo essa assistência.

Constatou-se a predominância de pessoas da faixa etária de 60 a 64 anos, seguido do grupo de 65 a 69 anos, na busca de assistência. Chama a atenção o fato de idosos com mais de 80 anos sendo atendidos nas UBS, o que mostra a longevidade da cidade e busca pela manutenção da saúde.

Tais achados corroboram com os estudos de Veras (2009) quanto ao aumento da procura pela saúde e manutenção da mesma pelas pessoas idosas que parecem demonstrar um cuidado mais minucioso pautado na prevenção, o que, certamente se converte não apenas em benefício próprio mas na saúde econômica do Estado.

\section{Reconhecimento e Agradecimentos}

A Capes, fomentadora que possibilitou a realização deste estudo e a Universidade de Passo Fundo pela Bolsa Institucional UPF, que possibilita o desenvolvimento da Pós-Graduação Stricto Sensu em Envelhecimento Humano. 


\title{
Cartography of the health of the elderly attended in basic units of public health
}

\author{
Abstract
}

With the aging population may increase the incidence of chronic diseases and seek health care. The objective of this study was to verify the health conditions of elderly people attending public health units in the city of Getúlio Vargas-RS. Diagnostic data were collected in the reports of care (e-SUS) of all Basic Health Unit (UBS, $n=5$ ), from January 1 to July 30, 2018. There were 8,634 consultations, 8,524 in the UBS and 110 at home. The prevalence of the International Classification of Diseases (ICD), General Medical Examination $(\mathrm{Z}=000, \mathrm{n}=1,144)$, Primary Essential Hypertension $(110, \mathrm{n}=439)$ and Blood Pressure Exams $(Z 013, \mathrm{n}=$ 169) was observed. The prevalence of care occurred in women $(n=5,309)$ in the age group of 60 to 64 years. Special attention should be given to routine consultations and the high incidence of chronic diseases related to blood pressure, especially in women, in public health care policies.

Keywords: Diagnosis; Report; Public health; Seniors; Diseases.

\section{Referências}

AGONDI, R.C., RIZZO, L.V., KALIL, J., et al. Imunossenescência. Rev. bras. Alerg. Imunopatol. v 35. n. 5, pp 169-175, 2012.

BOTTON, A., CUNICO, S.D., STREY, M.N. Diferenças de gênero no acesso aos serviços de saúde: problematizações necessárias. Mudanças - Psicologia da Saúde, 25 v.1, Jan.-Jun, pp. 67-72, 2017.

BRASIL. Lei n 8.080 de 19 de setembro de 1990. Brasília, DF, setembro de 1990.

INSTITUTO BRASILEIRO DE GEORGAFIA E ESTATÍSTICA (IBGE). Brasil em síntese, 2017. Disponível em https://cidades.ibge.gov.br/brasil/rs/getulio-vargas/panorama. Acesso em 12 de setembro de 2018.

INSTITUTO BRASILEIRO DE GEORGAFIA E ESTATÍSTICA (IBGE). Número de idosos cresce $18 \%$ em 5 anos e ultrapassa 30 milhões em 2017. Estatísticas Sociais, 2018. Disponível em: <https://agenciadenoticias.ibge.gov. br/agencia-noticias/2012-agencia-de-noticias/noticias/20980-numero-de-idosos-cresce-18-em-5-anos-e-ultrapassa30-milhoes-em-2017.html> Acesso em 07 de setembro de 2018.

ORGANIZAÇÃO MUNDIAL DA SAÚDE (OMS). Relatório Mundial de Envelhecimento e Saúde, 2015. Disponível em: <https://sbgg.org.br//wp-content/uploads/2015/10/OMS-ENVELHECIMENTO-2015-port.pdf> Acesso em 12 de setembro de 2018.

SANTOS, E.C., COUTO, B.M., BASTONE, A.C. Fatores associados à autoavaliação negativa da saúde em idosos cadastrados nas Unidades Básicas de Saúde. ABCS Health Sci.; 43 v.1 pp. 47-54, 2018.

VERAS, R. Envelhecimento populacional contemporâneo: demandas, desafios e inovações. Rev Saúde Pública; 43 v.3, pp. 548-54, 2009. 